\title{
Effect of magnetic field on electron spectrum in spherical nano-structures
}

\author{
V. Holovatsky;, O. Voitsekhivska, I. Bernik \\ Chernivtsi National University, 2 Kotsiubynsky St., 58012 Chernivtsi, Ukraine
}

Received September 28, 2013, in final form November 3, 2013

\begin{abstract}
The effect of a magnetic field on the energy spectrum and on the wave functions of an electron in spherical nano-structures such as single quantum dot and spherical layer is investigated. It is shown that the magnetic field removes the spectrum degeneration with respect to the magnetic quantum number. An increasing magnetic field induction entails a monotonous character of electron energy for the states with $m \geqslant 0$ and a nonmonotonous one for the states with $m<0$. The electron wave functions of the ground state and several excited states are studied considering the effect of the magnetic field. It is shown that $1 s$ and $1 p$ states are degenerated in the spherical layer driven by a strong magnetic field. In the limit case, a series of size-quantized levels produce the Landau levels which are typical of bulk crystals.
\end{abstract}

Key words: electron spectrum, quantum dot, spherical layer, magnetic field

PACS: 73.21.La, 73.21.-b

\section{Introduction}

The multilayered spherical nano-structures consisting of a core and a few spherical shells attract a particular attention of scientists. These structures are grown using the chemical colloidal method on the basis of CdS, CdSe, ZnS, ZnSe, HgS and other semiconductor materials [1-3]. Multilayered nano-structures have wide prospects for being utilized in medicine and electronics, for instance for the purpose of fabricating efficient biosensors and fluorescent labels [4, 5]. Multilayered spherical nano-structure containing two quantum wells formed by the core and spherical layer is called quantum-dot-quantum-well (QDQW). Such structures can be the basis for modern highly efficient white light sources [6]. In QDQW, a quasiparticle can be located in one of the quantum wells. The peculiarities of electron and hole location in multilayered spherical nano-structures have been theoretically studied in [7-10]. The impurities, external electrical and magnetic fields produce an effect on the location of quasi-particles in quantum wells [11-19] and consequently effect the optical properties of the structures. The on-center Coulomb impurity does not violate the spherical symmetry of the system and thus the Schrödinger equation for an electron or hole is solved exactly [11-13]. When the external fields are present, the spherical symmetry is violated and the calculation of an energy spectrum becomes more complicated due to the fitting conditions at the interfaces [14-16]. Therefore, in the majority of theoretical studies, the investigation of ground state energies of quasi-particles are performed within the variational method in the frames of the model of infinitely deep potential well [17, 18]. The effect of a magnetic field on the energies and on wave functions of the excited states of quasi-particles is still insufficiently investigated for a spherical quantum dot. The analogous problem for QDQW has not been studied at all.

In the case of high potential barrier of QDQW, the electron in low states does not penetrate through the interfaces of the system and quantum wells become decoupled [20]. The effect of a magnetic field on the states of electron located in the core or in the spherical layer of QDQW is different and can be investigated considering these two potential wells independently. Therefore, in this paper we study the

\footnotetext{
*E-mail: ktf@chnu.edu.ua
} 
magnetic field effect on the energy spectrum and on the wave functions of an electron located in the quantum dot (QD) and in the spherical layer (SL), assuming that the potential barriers are infinite.

\section{Schrödinger equation for the electron in spherical nano-structures driven by magnetic field}

We consider the spherical QD with the radius $r_{0}$ and the spherical layer with the inner and outer radii $r_{1}$ and $r_{2}$, respectively, having impenetrable boundaries. The coordinate system is taken in such a way that its origin is in the center of the structure and $\mathrm{Oz}$ axis coincides with the direction of the magnetic field induction.

The potentials of size quantization for the electron are as follows:

$$
\begin{gathered}
U^{(0)}(r)= \begin{cases}0, & r \leqslant r_{0}, \\
\infty, & r>r_{0},\end{cases} \\
U^{(1)}(r)= \begin{cases}0, & r_{1} \leqslant r \leqslant r_{2}, \\
\infty, & r<r_{1}, r>r_{2} .\end{cases}
\end{gathered}
$$

Here, $U^{(0)}$ is the potential energy of an electron in spherical QD and $U^{(1)}$ - in SL having impenetrable boundaries.

Schrödinger equations for the electron in these systems in a magnetic field are as follows:

$$
\left[\frac{1}{2 \mu}\left(\vec{p}-\frac{e}{c} \vec{A}\right)^{2}+U^{(0,1)}(r)\right] \psi^{(0,1)}(\vec{r})=E^{(0,1)} \psi^{(0,1)}(\vec{r}) .
$$

When $\vec{A}=[\vec{r} \times \vec{B}] / 2$, the Hamiltonians become

$$
H^{(0,1)}=-\frac{\hbar^{2}}{2 \mu} \Delta+\frac{e B}{2 c \mu} L_{z}+\frac{e^{2} B^{2} r^{2} \sin ^{2} \theta}{8 c^{2} \mu}+U^{(0,1)}(r),
$$

where $L_{z}=-\mathrm{i} \hbar \partial / \partial \varphi$.

Using the dimensionless magnitudes: $R^{*}=e^{2} /\left(2 \varepsilon a^{*}\right)$ - effective Rydberg energy, $a^{*}=\hbar^{2} \varepsilon /\left(\mu e^{2}\right)-$ effective Bohr radius and parameter $\eta=\hbar \omega_{\mathrm{c}} /\left(2 R^{*}\right)$, where $\omega_{\mathrm{c}}=B e /(\mu c)-$ cyclotron frequency, the Hamiltonian (4) is transformed into

$$
H^{(0,1)}=-\Delta+\eta L_{z}+\frac{1}{4}(\eta r \sin \theta)^{2}+U^{(0,1)}(r)
$$

When $\eta=0$, the Schrödinger equation with Hamiltonian (5) has the exact solutions

$$
\Phi_{n l m}^{(0,1)}(r, \theta, \varphi)=R_{n l}^{(0,1)}(r) Y_{l m}(\theta, \varphi),
$$

where $R_{n l}^{(0)}(r)=A_{n l}^{(0)} j_{l}\left(\chi_{n l} r / r_{0}\right), R_{n l}^{(1)}(r)=A_{n l}^{(1)} j_{l}\left(k_{n l} r\right)+B_{n l}^{(1)} n_{l}\left(k_{n l} r\right), A_{n l}^{(0)}=\sqrt{2} /\left[r_{0}^{3 / 2} j_{l+1}\left(\chi_{n l}\right)\right], B_{n l}^{(1)}=$ $-A_{n l}^{(1)} j_{l}\left(k_{n l} r_{1}\right) / n_{l}\left(k_{n l} r_{1}\right), j_{l}(z), n_{l}(z)$ are Bessel spherical functions of the first and the second kind, respectively, $\chi_{n l}$ are the roots of Bessel spherical function $\left[j_{l}\left(\chi_{n l}\right)=0\right.$ ], the values $k_{n l}$ are fixed by the condition $R_{n l}^{(1)}\left(r_{2}\right)=0$ and the coefficients $A_{n l}^{(1)}$ are fixed by the normality condition.

The square term (with respect to the magnetic field) in the Hamiltonian (5) rapidly increases its contribution into the complete energy. Thus, it is impossible to use the perturbation method. In order to obtain the ground state energy using the variational method it is necessary to define the approximated wave function. In [21] it is written as follows:

$$
\psi_{100}^{(0,1)}(\vec{r})=\mathrm{e}^{-g(\vec{r})} \Phi_{100}^{(0,1)}(\vec{r})
$$

where the function $g(\vec{r})$ is to ensure the compensation of a quadratic term after substitution of (7) into (5). The minimum condition for

$$
F(\eta)=\frac{1}{4}(\eta r \sin \theta)^{2}-|\vec{\nabla} g(\vec{r})|^{2}+\Delta g(\vec{r})
$$


at $\left.g(\vec{r})\right|_{\eta \rightarrow 0}=0$ fixes $g(\vec{r})=\eta r^{2} \sin ^{2} \theta / 4$. Herein, $F(\eta)$ linearly depends on $\eta$, thus, the variational function of the electron ground state can be written as follows:

$$
\psi_{100}^{(0,1)}(\vec{r})=C \exp \left(-\eta r^{2} \sin ^{2} \theta / 4\right) \Phi_{100}^{(0,1)}(\vec{r}) \exp (\lambda r)
$$

where $C$ is the normality constant and $\lambda$ is the variational parameter. The form of the variational wave function is confirmed by physical considerations. The magnetic field directed along $\mathrm{Oz}$ axis deforms the wave function compressing it in perpendicular direction. This fact is represented by an angular dependence of the function (9). Variational parameter $\lambda$ and the energy of ground state are obtained from the minimum of the whole energy

$$
E_{100}^{(0,1)}=\min _{\lambda}\left\langle\psi_{100}^{(0,1)}(\vec{r})\left|H^{(0,1)}\right| \psi_{100}^{(0,1) *}(\vec{r})\right\rangle .
$$

In order to study the excited states, the orthonormality condition for the wave functions should be fulfilled, which makes the problem rather complicated. Therefore, we are going to use another method to solve it. We expand the wave function using a complete set of eigenfunctions of the electron in a spherical nano-structure without the magnetic field obtained as the exact solutions of Schrödinger equation [15]. When the magnetic field is applied, the spherical symmetry is violated and the orbital quantum number becomes inconvenient. The new states characterized by a magnetic quantum number $m$ are presented as a linear combination of the states $\Phi_{n l m}^{(0,1)}(\vec{r})$

$$
\psi_{j m}^{(0,1)}(\vec{r})=\sum_{n} \sum_{l} c_{n l} \Phi_{n l m}^{(0,1)}(\vec{r})
$$

Substituting (11) into Schrödinger equation with Hamiltonian (5), we obtain a secular equation for the electron energy spectrum

$$
\left|H_{n l, n^{\prime} l^{\prime}}^{(0,1)}-E_{j m}^{(0,1)} \delta_{n, n^{\prime}} \delta_{l, l^{\prime}}\right|=0
$$

where the matrix elements $H_{n^{\prime} l^{\prime}, n l}^{(0,1)}$ have the form

$$
\begin{aligned}
H_{n^{\prime} l^{\prime}, n l}^{(0,1)} & =\left(E_{n l}^{0(0,1)}+m \eta\right) \delta_{n^{\prime} n} \delta_{l^{\prime} l}+\frac{\eta^{2}}{2}\left\{\alpha_{l, m} \delta_{l^{\prime}, l+2}+\beta_{l, m} \delta_{l^{\prime}, l}+\gamma_{l, m} \delta_{l^{\prime}, l-2}\right\} I_{n^{\prime} l^{\prime}, n l}^{(0,1)}, \\
I_{n^{\prime} l^{\prime}, n l}^{(0)} & =A_{n^{\prime} l^{\prime}}^{(0)} A_{n l}^{(0)} \int_{0}^{r_{0}} r^{4} j_{l^{\prime}}\left(\chi_{n^{\prime} l^{\prime}} r / r_{0}\right) j_{l}\left(\chi_{n l} r / r_{0}\right) \mathrm{d} r, \\
I_{n^{\prime} l^{\prime}, n l}^{(1)} & =\int_{r_{1}}^{r_{2}} r^{4}\left[A_{n^{\prime} l^{\prime}} j_{l^{\prime}}\left(k_{n^{\prime} l^{\prime}} r\right)+B_{n^{\prime} l^{\prime}} n_{l^{\prime}}\left(k_{n^{\prime} l^{\prime}} r\right)\right]\left[A_{n l} j_{l}\left(k_{n l} r\right)+B_{n l} n_{l}\left(k_{n l} r\right)\right] \mathrm{d} r, \\
\alpha_{l, m} & =-\sqrt{\frac{\left[(l+2)^{2}-m^{2}\right]\left[(l+1)^{2}-m^{2}\right]}{(2 l+5)(2 l+3)^{2}(2 l+1)}}, \quad \gamma_{l, m}=-\sqrt{\frac{\left(l^{2}-m^{2}\right)\left[(l-1)^{2}-m^{2}\right]}{(2 l+1)(2 l-1)^{2}(2 l-3)}} \\
\beta_{l, m} & =1-\frac{(l+1)^{2}-m^{2}}{(2 l+1)(2 l+3)}-\frac{l^{2}-m^{2}}{4 l^{2}-1}, \quad
\end{aligned}
$$

Using the eigenvalues and eigenvectors of the matrix

$$
F_{n l, n^{\prime} l^{\prime}}^{(0,1)}=H_{n l, n^{\prime} l^{\prime}}^{(0,1)}-E_{j m}^{(0,1)} \delta_{n, n^{\prime}} \delta_{l, l^{\prime}},
$$

we obtain the energy spectrum and wave functions of the electron in a spherical nano-structure driven by the magnetic field. 
Table 1. Expansion coefficients of electron wave functions $\psi_{100}^{(0)}, \psi_{110}^{(0)}, \psi_{11-1}^{(0)}$ in QD and $\psi_{100}^{(1)}, \psi_{110}^{(1)}, \psi_{11-1}^{(1)}$ in SL at $B=40 \mathrm{~T}$.

\begin{tabular}{|c||c|c|c|c|c|c|}
\hline & $\psi_{100}^{(0)}$ & $\psi_{100}^{(1)}$ & $\psi_{110}^{(0)}$ & $\psi_{110}^{(1)}$ & $\psi_{11-1}^{(0)}$ & $\psi_{11-1}^{(1)}$ \\
\hline \hline$c_{10}$ & 0.9986 & 0.7018 & - & - & - & - \\
\hline$c_{11}$ & - & - & 0.9993 & 0.9151 & 0.9991 & 0.7949 \\
\hline$c_{12}$ & 0.0378 & 0.6888 & - & - & - & - \\
\hline$c_{13}$ & - & - & 0.0336 & 0.3969 & 0.0282 & 0.5923 \\
\hline$c_{14}$ & 0.0007 & 0.1801 & - & - & - & - \\
\hline$c_{15}$ & - & - & 0.0006 & 0.0705 & 0.0005 & 0.1306 \\
\hline$c_{16}$ & 0 & 0.0232 & - & - & - & - \\
\hline$c_{17}$ & - & - & 0 & 0,0069 & 0 & 0.0144 \\
\hline$c_{20}$ & 0.0360 & 0.0044 & - & - & - & - \\
\hline$c_{21}$ & - & - & 0.0161 & 0.0044 & 0.0331 & 0.0087 \\
\hline$c_{22}$ & 0.0009 & 0.0014 & - & - & - & - \\
\hline$c_{23}$ & - & - & 0.0010 & 0.0007 & 0.0007 & 0.0022 \\
\hline$\sum_{i} c_{i}^{2}$ & 1.000 & 1.000 & 1.000 & 1.000 & 1.000 & 1.000 \\
\hline
\end{tabular}

\section{Analysis of the results}

The computer calculations were performed using the physical parameters of CdSe semiconductor material: electron effective mass $\mu=0.13 m_{e}$ ( $m_{e}-$ the mass of pure electron), dielectric constant $\varepsilon=$ 10.6 .

Expanding the wave functions (13) we took into account a sufficient number of terms, provided that the sum of squares of expansion coefficients was equal to a unit with the accuracy not less than $0.01 \%$. In table 1 the expansion coefficients for the lowest states at $B=40 \mathrm{~T}$ are presented. Here one can see that the required accuracy is provided by 6 major terms. For convenience, we use the same quantum numbers characterizing the states of an electron in a nano-structure driven by a magnetic field as the ones without the field.

The dependencies of electron energy spectrum on the magnetic field induction in CdSe QD and SL are presented in figure 1

From figure 1 one can see that the energy of $\psi_{100}$ state calculated by a variational method correlates well to the one obtained by the matrix method. Moreover, even in the case of a strong magnetic field, the

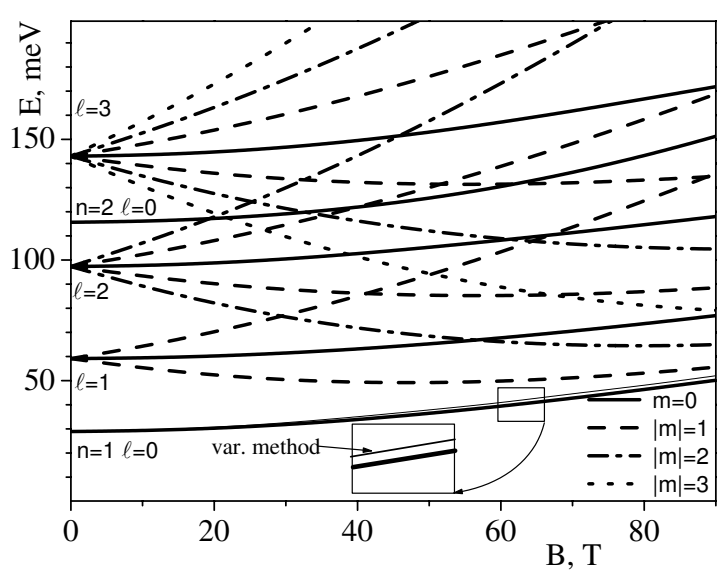

(a)

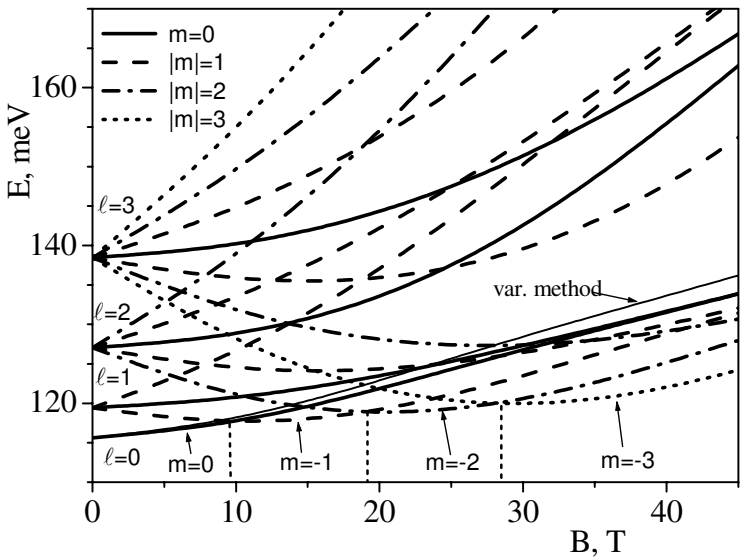

(b)

Figure 1. Electron energy spectrum as function of magnetic field induction in QD with $r_{0}=10 \mathrm{~nm}$ (a) and SL with $r_{1}=10 \mathrm{~nm}, r_{2}=15 \mathrm{~nm}(\mathrm{~b})$. 
(a)

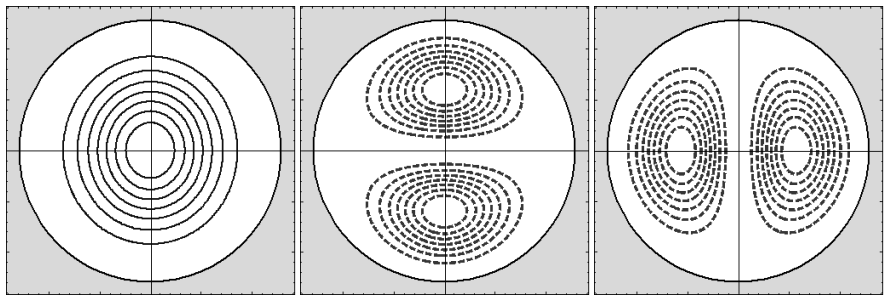

(b)

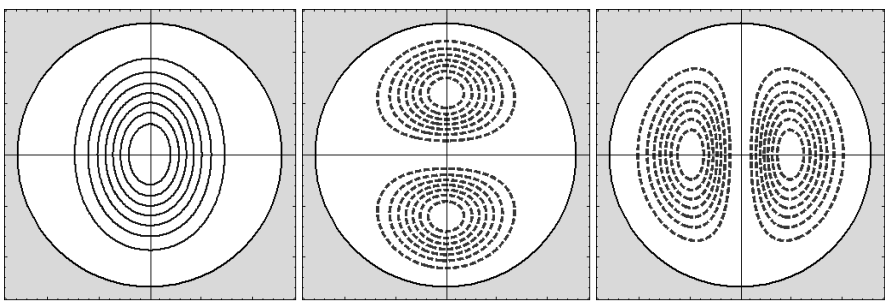

Figure 2. Distribution of probability density of electron location in QD with $r_{0}=10 \mathrm{~nm}$, at $B=40 \mathrm{~T}$ (a), $B=80 \mathrm{~T}(\mathrm{~b})$ for the quantum states with $\psi_{100}^{(0)}, \psi_{110}^{(0)}, \psi_{11-1}^{(0)}$.

error for the electron energy in both nano-structures does not exceed 3\%. Comparing the dependencies shown in figures 1(a) and1(b), one can see that the magnetic field produces a greater effect on the energy states of an electron located in SL than on the energy states in QD. In both nano-structures, the degeneracy over the magnetic quantum number is removed. The energies of the states with $m \geqslant 0$ increase under the effect of the magnetic field. For the states with $m<0$, the non-monotonous dependence of the energy on the magnetic field is caused by the linear and quadratic terms contributed by the magnetic field into the Hamiltonian (7).

(a)
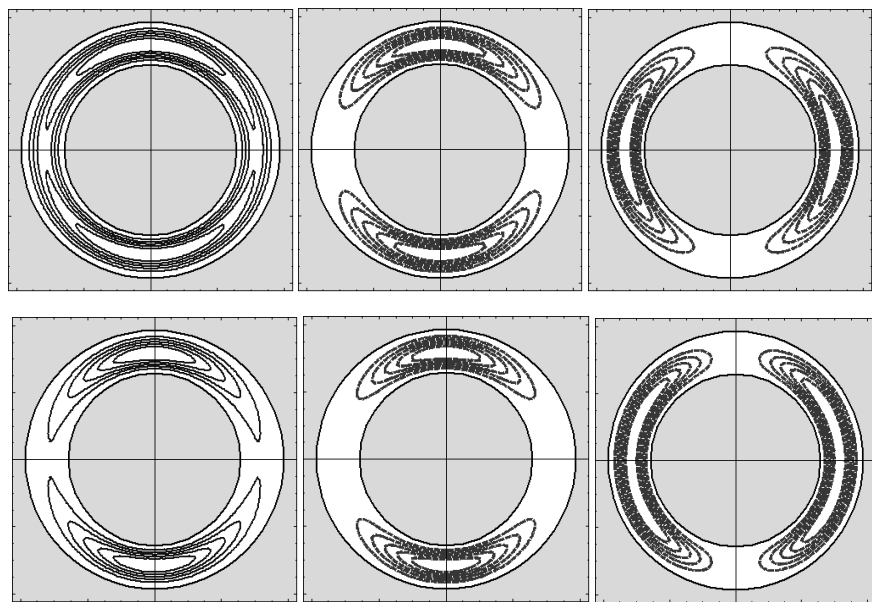

(b)

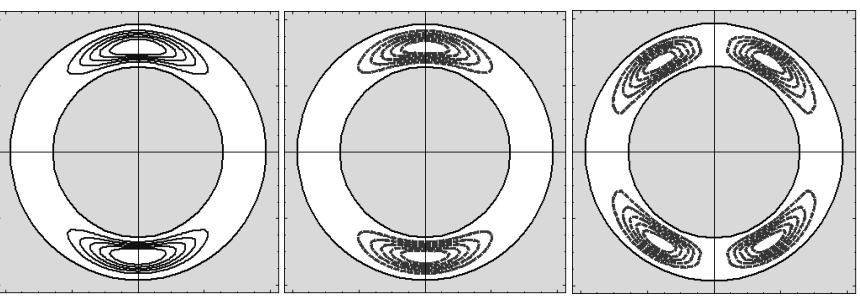

(c)

Figure 3. Distribution of probability density of electron location in SL with $r_{1}=10 \mathrm{~nm}, r_{2}=15 \mathrm{~nm}$ at $B=10 \mathrm{~T}(\mathrm{a}), B=20 \mathrm{~T}(\mathrm{~b})$ and $B=40 \mathrm{~T}$ (c) for the quantum states with $\psi_{100}^{(1)}, \psi_{110}^{(1)}, \psi_{11-1}^{(1)}$. 
In the SL placed into a strong magnetic field, $1 s$ and $1 p$ states with $m=0$ are degenerated, unlike in the QD. However, in zero-dimensional systems, the electron ground state is always non-degenerated. Consequently, when the magnetic field increases, the lowest states with $m=0,-1,-2, \ldots$ successively play the role of the ground state. The ground state of an electron with a certain value of a magnetic quantum number transforms into the state with the other $m$ when the magnetic field intensity increases at an equal magnitude. The distance between the points of such a transition increases when SL radius becomes smaller. A similar behavior of the electron ground state energy was theoretically obtained and experimentally confirmed for the semiconductor quantum rings [22]. The oscillation of energies is known as Aharonov Bohm effect.

The distribution of probability density of electron location in QD and SL in different quantum states is presented in figures 2,3 respectively.
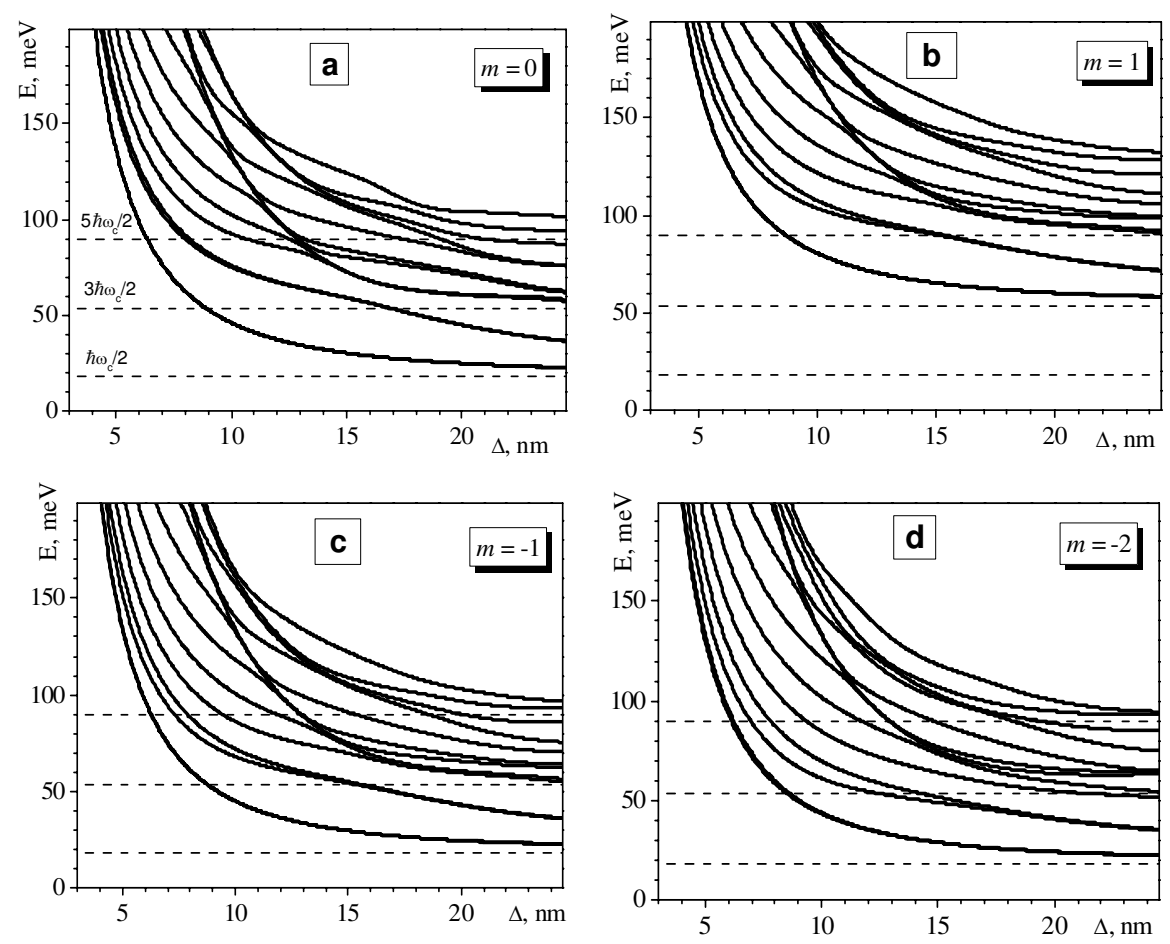

Figure 4. Electron energy spectrum as function of $\Delta=r_{2}-r_{1}$ at $B=40 \mathrm{~T}$ for $m=0$ (a), $m=1$ (b), $m=-1$ (c) and $m=-2$ (d). Landau levels in bulk crystal are shown by dash lines.

Figures 23, prove that the electron wave functions are deformed due to the effect of the strong magnetic field. When its induction increases, the angular probability increases near $\theta=0, \pi$ and decreases near $\theta=\pi / 2$. Herein, in the SL the wave function $\psi_{100}^{(1)}$ of $1 s$ state, due to the deformation, becomes similar to the $\psi_{110}^{(1)}$ one of the excited $1 p$ state. At $B=40 \mathrm{~T}$, these states become indistinguishable both for the distribution of probability density [figure 3(c)] and the energy [figure 1(b)].

Computer calculations prove that in the limit case when the inner radius of SL $r_{1}$ diminishes at $r_{2}=$ const and at the constant magnetic field induction, the electron energy spectrum coincides with the one for the QD with $r_{0}=r_{2}$.

In limit cases, when the QD radius or SL thickness increases at $B=$ const due to a decrease of the quantum confining effect, the electron energy levels should coincide with Landau levels which are typical of a bulk crystal placed into the magnetic field. The process of the formation of Landau levels for the quantum states with $m=0,1,-1$ in the SL is presented in figure 4 .

Figure 4proves that the quantum confining effect diminishes when the sizes of the structure increase. This process is accompanied by a decrease of all energy levels and by the formation of Landau levels. For example, at $m=0$, the lowest Landau level is formed by the set of levels with $n=1$, the next one with 
$n=2$ and so on. A complicated dependence of an energy spectrum is observed during their formation due to the anti-crossing effect.

\section{Summary}

We studied the electron energy spectrum in QD and SL under the effect of a magnetic field. The problem is solved using the variational method and the method of electron wave function expansion over the set of eigenfunctions being the exact solutions of Schrödinger equation for the same structures without the magnetic field. The results obtained within the both methods are in good agreement. The variational method describes the lowest electron state with $m=0$. However, the ground state in SL is formed by the states with $m=0,-1,-2, \ldots$ consequently with the growth of the magnetic field induction. It is shown that the major contribution into the expansion of a wave function of an arbitrary electron state even in the strong magnetic field is performed by a few neighboring (over the energy) quantum states which are the exact solutions of Schrödinger equation for the electron when there is no magnetic field.

The wave functions of the electron in QD and SL are deformed under the effect of a magnetic field. The degeneration of an energy spectrum with respect to a magnetic quantum number is removed. The electron energies for the states with positive and negative values of a magnetic quantum number differently depend on the magnetic field induction: for the states with $m \geqslant 0$, the energy monotonously increases and for $m<0$, the energy decreases at first and then, only when the magnetic field becomes strong enough, enhances.

It is proven that the effect of a magnetic field on the electron energy spectrum in SL is stronger than that in QD. Moreover, it is shown that in SL driven by the strong magnetic field, the neighboring states with the same $m$ become degenerated. For example, in the studied structure, $1 s$ and $1 p$ states are degenerated at $B>30 \mathrm{~T}$. The degeneration of higher energy states takes place at a stronger magnetic field.

The validity of the obtained results is confirmed by the limit cases: when the quantum confining effect reduces at a constant magnetic field induction, the energy levels rebuild, saturate and form the respective Landau levels. When the inner radius of SL $\left(r_{1}\right)$ decreases at $r_{2}=$ const, the electron energy spectrum becomes the same as in the QD having the radius $r_{2}$.

The results of the investigation make it possible to estimate the energies and the most probable place of electron location in QDQW. Different dependencies of electron energies in QD and SL on the magnetic field induction lead to their anticrossing in QDQW. This feature permits to change the quasiparticle location by a magnetic field for multilayered systems with penetrable interfaces.

\section{References}

1. Eychmuller A., Mews A., Weller H., Chem. Phys. Lett., 1993, 208, 59; doi 10.1016/0009-2614(93)80076-2

2. Dorfs D., Eychmuller A., Z. Phys. Chem., 2006, 220, 1539; doi 10.1524/zpch.2006.220.12.1539

3. Little R., El-Sayed M., Bryant G., Burke S., Chem. Phys., 2001, 114, 1813.

4. Frasco M.F., Chaniotakis N., Sensors, 2009, 9, 7266; doi 10.3390/s90907266.

5. Liu Y.S., Sun Y., Vernier P.T., Liang C. H., Chong S.Y., Gundersen M.A., J. Phys. Chem. C. Nanomater Interfaces, 2007, 111, 2872; doi 10.1021/jp0654718

6. Demir H., Nizamoglu S., Mutlugun E., Ozel T., Sapra S., Gaponik N., Eychmuller A., Nanotechnology, 2008, 19, 335203; doi 10.1063/1.2898892

7. SalmanOgli A., Rostami A., J. Nanopart. Res., 2011, 13, 1197; doi 10.1007/s11051-010-0112-2

8. Tkach N., Voitsekhovska O., Holovatsky V., Mihalyova M., Izvestiya vuzov. Fizika, 1998, 12, 58 (in Russian) [Russ. Phys. J., 1998, 41, 1229; doi 10.1007/BF02514561.

9. Holovatsky V., J. Phys. Stud., 1998, 2, 583 (in Ukrainian).

10. Tkach M., Holovatsky V., Voitsekhivska O., Mikhalyova M., Electrochemical Society Proceedings, 1998, 25, 316.

11. Tkach M., Holovatsky V., Voitsekhivska O., Fiz. Teh. Pol., 2000, 34, 602 (in Russian) [Semiconductors, 2000, 34, 583; doi 10.1134/1.1188032.

12. Holovatsky V., Makhanets O., Voitsekhivska O., Physica E, 2009, 41, 1522; doi 10.1016/j.physe.2009.04.027

13. Boichuk V.I., Bilynskyi I.V., Leshko R.Ya., Voronyak L.Ya., Ukr. J. Phys., 2009, 54, 1021.

14. Tas H., Sahin M., J. Appl. Phys., 2012, 111, 083702; doi 10.1063/1.4751483

15. Wu S., Wan L., J. Appl. Phys., 2012, 111, 063711; doi 10.1063/1.3695454. 
16. Rahmani K., Zorkani I., M. J. Condensed Matter., 2009, 11, 35.

17. Chakraborty T., Apalkov V., Physica E, 2003, 16, 253; doi 10.1016/S1386-9477(02)00674-4

18. Xiao Z., J. Appl. Phys., 1999, 86, 4509; doi 10.1063/1.371394

19. Planelles J., Diaz J., Climente J., Jaskolski W., Phys. Rev. B, 2002, 65, 245302; doi 10.1103/PhysRevB.65.245302

20. Battaglia D., Blackman B., Peng X., J. Am. Chem. Soc., 2005, 127, 10889-10897; doi 10.1021/ja0437297.

21. Jiang H., Phys. Rev. B., 1987, 35, 9287; doi 10.1103/PhysRevB.35.9287

22. Lorke A., Luyken R., Govorov A., Kotthaus J.J., Garcia M., Petroff P., Phys. Rev. B, 2000, 84, 2223; doi 10.1103/PhysRevLett.84.2223.

\title{
Вплив магнітного поля на спектр електрона у сферичних наноструктурах
}

\author{
В.А. Головацький, О.М. Войцехівська, І.Б. Бернік \\ Чернівецький національний університет ім. Юрія Федьковича, вул. Коцюбинського, 2, 58012 Чернівці, \\ Україна
}

Досліджено вплив постійного магнітного поля на енергетичний спектр та хвильові функції електрона в сферичних наноструктурах: простій квантовій точці та сферичній плівці. Показано, що під дією магнітного поля знімається виродження енергетичного спектру за магнітним квантовим числом. Збільшення індукції магнітного поля приводить до монотонного зростання енергії станів з магнітним квантовим числом $m \geqslant 0$ та немонотонної залежності енергії станів з $m<0$. Досліджено вплив магнітного поля на хвильові функції основного та декількох збуджених станів електрона. У сферичній плівці зі збільшенням індукції магнітного поля відбувається виродження $1 s$ та $1 p$ станів. При збільшенні розмірів наносистеми чи збільшенні індукції магнітного поля відбувається формування серій рівнів Ландау, що характерні для масивного кристалу.

Ключові слова: спектр електрона, квантова точка, сферична плівка, магнітне поле 